Article

\title{
Evaluation of the RPC Model for Ziyuan-3 Three-line Array Imagery
}

\author{
Zhonghua Hong ${ }^{1, *}$, Shengyuan $\mathrm{Xu}{ }^{1}$, Yun Zhang ${ }^{1, *}$, Yanling Han ${ }^{1}$ and Yongjiu Feng ${ }^{2}$ \\ 1 College of Information Technology, Shanghai Ocean University, 999 Hu-Chenghuan Road, Shanghai 201306, China \\ 2 College of Marine Science, Shanghai Ocean University, 999 Hu-Chenghuan Road, Shanghai 201306, China \\ * Correspondence to: zhhong@shou.edu.cn (Z.H.); y-zhang@shou.edu.cn (Y.Z.)
}

\begin{abstract}
Ziyuan-3 (ZY-3) satellite is the first civilian stereo mapping satellite in China and was designed to achieve the 1:50000 scale mapping for land and ocean. Rigorous sensor model (RSM) is required to build the relationship between the three-dimensional (3D) object space and two-dimensional (2D) image space of ZY-3 satellite imagery. However, each satellite sensor has its own imaging system with different physical sensor models, which increase the difficulty of geometric integration of multi-source images with different sensor models. Therefore, it is critical to generate generic model especially rational polynomial coefficients (RPCs) of optical imagery. Recently, relatively a few researches have been conducted on RPCs generation to ZY-3 satellite. This paper proposes an approach to generate the RPCs for ZY-3 imagery from RSM. Three scenarios experiments with different terrain features (such as ocean, city and grassland) are designed and conducted to comprehensively evaluate the replacement accuracies of this approach and analyze the RPCs fitting error. All the experimental results demonstrate that the RPCs is suitable for ZY-3 imagery and can be used as a replacement for the RSM of ZY-3 imagery.
\end{abstract}

Keywords: Rational Polynomial Coefficients (RPCs); Ziyuan-3 satellite; Rigorous sensor model (RSM)

\section{Introduction}

High resolution satellite imagery (HRSI) becomes a large area source of information. Remote sensing technology has played a more and more important role in Earth observation. Sensor model of HRSI is crucial to land and ocean mapping, which is typically divided into two categories, namely the rigorous sensor models (RSM) and generalized models (GL) (Poli and Toutin, 2012). The RSM of space-borne linear array CCD optical imagery is mainly based on linear array co-linearity equation (Poli 2015; Jannati et al., 2017). However, each satellite sensor has its own imaging system with various RSM which increase the difficulty of developing geometric processing software that is capable of handling multi-source remote sensing data. For the aforementioned disadvantages, the development of replacement sensor models independent of sensor platforms and sensor types becomes attractive. Therefore, we need a generic model for processing of new satellite sensor is launched (Toutin 2004; Eftekhari et al., 2013). The rational function model (RFM) is the ratio of two cubic polynomials with 78 rational polynomial coefficients (RPCs) has been reported as an alternative sensor orientation model for high-resolution satellite imagery (HRSI) (Fraser et al., 2005), such as IKONOS, Quickbird, GeoEye-1 and WorldView-2, etc (Dial et al., 2003; Fraser et al., 2003, 2005, 2006, 2009; Li et al., 2007, 2008; Tong et al., 2010, 2012).

Recently, relatively a few researches have been conducted on RPCs generation to ZY-3 satellite. As a relatively new optical system, the Ziyuan-3 (ZY-3) satellite has been in operation since 9 January 2012. ZY-3 satellite is equipped with three-line scanners (nadir, backward, and forward views) and provides worldwide coverage being suitable for applications such as the creation of maps on a scale of 1:50000 and the updating of the maps on a 1:25000 scale (Chen et al., 2015, Tang et al., 2015).

This paper presents an approach to generate the RPCs for ZY-3 imagery from RSM. Three scenarios experiments with different terrain features (such as ocean, city and grassland) are designed and conducted to comprehensively evaluate the replacement accuracies of this approach and analyze the RPCs fitting error. 


\section{Study area and data sources}

We performed a comprehensive experiment to test the potential of RPC generation for ZY-3 imagery using three data sets in the ocean region, city region and grassland region, respectively. Figure 1 shows three study areas (Hulunbuir City, Shanghai City and Sansha City, China) and scenes with nadir (NAD), forward (FWD) and backward (BWD) images of ZY-3 satellite. Table 1 presents detailed information about these images used in the experiment, comprising the acquisition time, sensor name, image size, resolution, minimum/maximum elevation and center location.

Table 1. Image information of ZY-3 images

\begin{tabular}{|c|c|c|c|c|c|c|}
\hline Study area & $\begin{array}{l}\text { Acquisition } \\
\text { time }\end{array}$ & Sensors & $\begin{array}{c}\text { Image size } \\
\text { (line by } \\
\text { pixel) }\end{array}$ & $\begin{array}{l}\text { Resolution } \\
\text { (meter) }\end{array}$ & $\begin{array}{c}\text { Minimum and } \\
\text { maximum elevation } \\
\text { (meter) }\end{array}$ & Center Location \\
\hline \multirow{3}{*}{$\begin{array}{l}\text { Hulunbuir } \\
\text { city }\end{array}$} & \multirow{3}{*}{ 2013-06-04 } & FWD & 16384,16306 & 4.0 & \multirow{3}{*}{516,1037} & \multirow{3}{*}{$\begin{array}{l}\text { E } 118.88819^{\circ} \\
\text { N } 48.845417^{\circ}\end{array}$} \\
\hline & & NAD & 24576,24516 & 2.0 & & \\
\hline & & BWD & 16384,16306 & 4.0 & & \\
\hline \multirow{3}{*}{$\begin{array}{l}\text { Shanghai } \\
\text { city }\end{array}$} & \multirow{3}{*}{ 2013-07-10 } & FWD & 16384,16306 & 3.0 & \multirow{3}{*}{0,468} & \multirow{3}{*}{$\begin{array}{c}\text { E } 121.360634^{\circ} \\
\text { N } 31.08322^{\circ}\end{array}$} \\
\hline & & NAD & 24576,24516 & 2.0 & & \\
\hline & & BWD & 16384,16306 & 3.0 & & \\
\hline \multirow{3}{*}{ Sansha city } & \multirow{3}{*}{ 2013-05-01 } & FWD & 16384,16306 & 4.0 & \multirow{3}{*}{0,16} & \multirow{3}{*}{$\begin{array}{l}\text { E } 112.414345^{\circ} \\
\text { N } 16.768612^{\circ}\end{array}$} \\
\hline & & NAD & 24576,24516 & 2.0 & & \\
\hline & & BWD & 16384,16306 & 4.0 & & \\
\hline
\end{tabular}

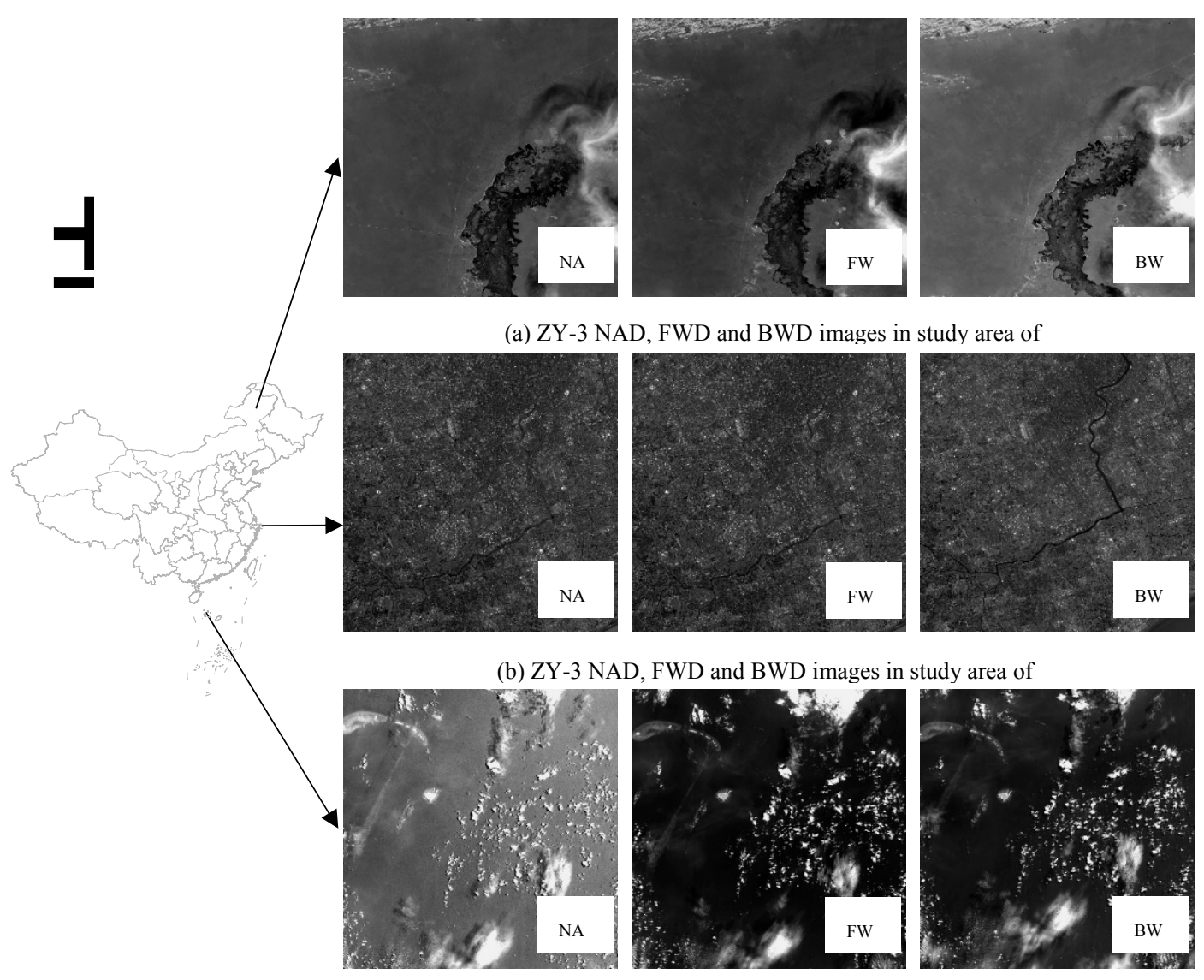

(c) ZY-3 NAD, FWD and BWD images in study area of

Figure 1. The three study areas and scenes with NAD, FWD and BWD images of ZY-3 satellite

\section{Methodology}

Figure 2 shows the entire framework for RPC generation of ZY-3 three-line array imageries. Four major issues are investigated: (1) to build RSM for ZY-3 imagery, (2) to generate virtual grid control points (VGCPs) 
generation based on RSM, (3) to estimate RPCs using CTPs selected from generated VGCPs, and (4) to evaluate the accuracy of RPCs generation by comparing the differences between the 2D image coordinates of CKP by RFM and RSM of CKPs. The methods are discussed in details in the following sections.

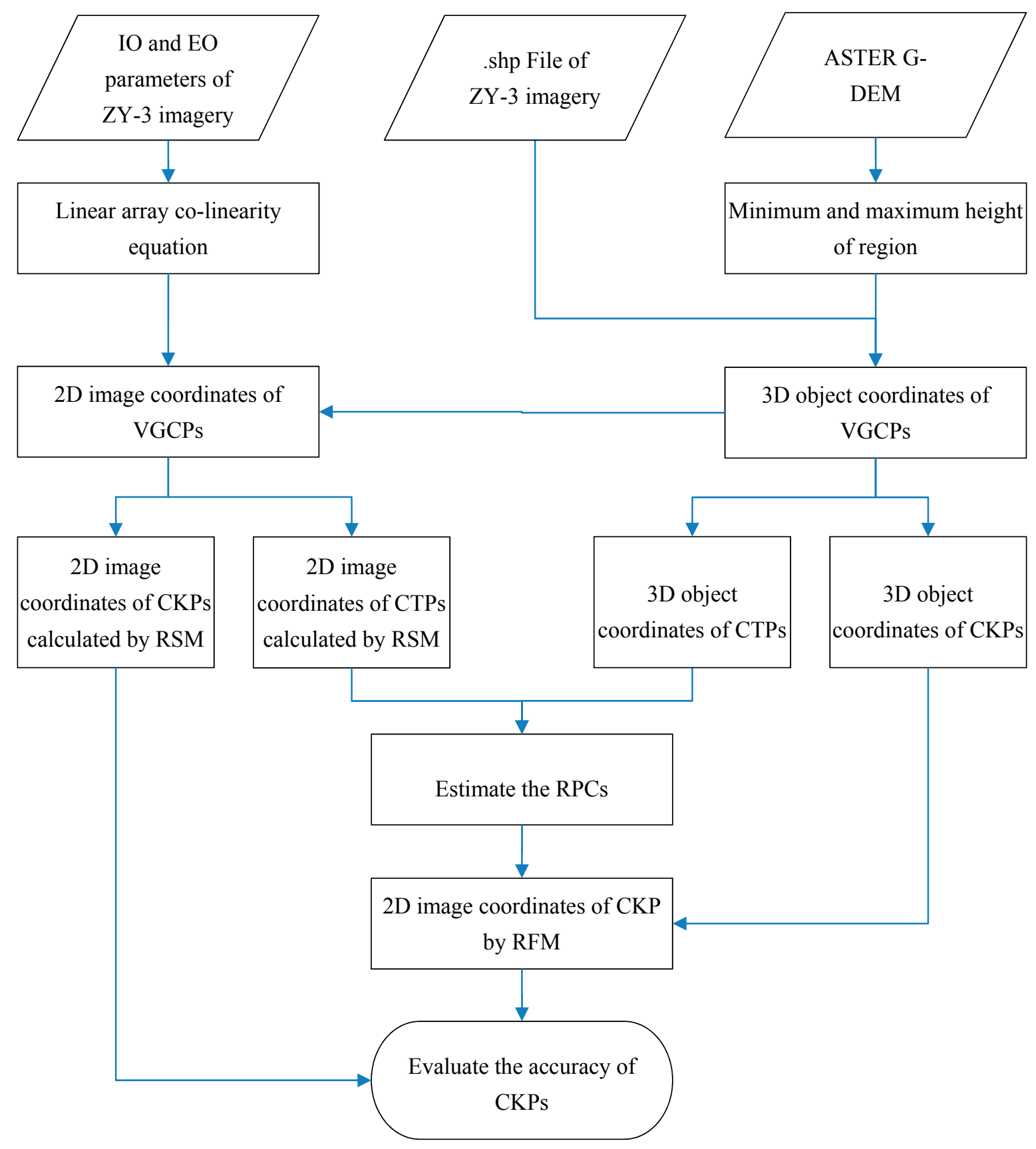

Figure 2. Framework for RPCs generation of ZY-3 imagery

\subsection{RSM of ZY-3 imagery based on linear array co-linearity equation}

The scanning mode of ZY-3 imagery is push-broom linear CCD, which is scanned along the flight direction (Tang et al., 2015).To assume that the flight direction as coordinates $\mathrm{x}$ direction, scan line direction as the coordinate y direction, the sensor model can be written as following:

$$
\left[\begin{array}{l}
X_{P} \\
Y_{P} \\
Z_{P}
\end{array}\right]_{W G S 84}=\left[\begin{array}{l}
X_{S}(t) \\
Y_{S}(t) \\
Z_{S}(t)
\end{array}\right]_{W G S 84}+m \cdot R_{J 2000}^{W G S 84}(t) \cdot R_{\text {Orbit }}^{J 2000}(t) \cdot R_{\text {Body }}^{\text {Orbit }} \cdot\left[\left[\begin{array}{l}
D_{x} \\
D_{y} \\
D_{z}
\end{array}\right]+\left[\begin{array}{l}
d_{x} \\
d_{y} \\
d_{z}
\end{array}\right]+R_{\text {Camera }}^{\text {Body }} \cdot f\left[\begin{array}{l}
\tan \left(\psi_{Y}\right) \\
\tan \left(\psi_{X}\right) \\
-1
\end{array}\right]\right]
$$


Where, $\left(X_{S}(t), Y_{S}(t), Z_{S}(t)\right)$ and $\left(X_{P}, Y_{P}, Z_{P}\right)$ are the perspective center coordinates and object coordinates in the WGS84 coordinate system, respectively. $m$ denotes the scaling factor, $f$ is the focal length of the satellite camera. ( $\left.R_{\text {Body }}^{\text {Orbit }}, R_{\text {Orbit }}^{J 2000}(t), R_{J 2000}^{W G S 84}(t)\right)$ are the rotation matrices from the satellite body system to satellite orbit system, J2000 coordinate system and WGS84 coordinate system, respectively. $\left(D_{x}, D_{y}, D_{z}\right)$ and $\left(d_{x}, d_{y}, d_{z}\right)$ are the displacement of the phase center and CCD array center of the satellite body, respectively. $R_{\text {Camera }}^{\text {Body }}$ represents the rotation matrix from the satellite camera coordinate system to the body coordinate system. $\left(\psi_{X}, \psi_{Y}\right)$ denotes the look angle. Meanwhile, $R_{\text {Camera }}^{\text {Body }}$ were measured before the satellite launch.

Rotation angles and perspective center coordinate can be interpolated using second-order polynomials with time $t$. Furthermore, the relationship between the look angle and image coordinates $(x, y)$ can be expressed as following:

$$
\left[\begin{array}{l}
x-x_{0} \\
y-y_{0} \\
-f
\end{array}\right]=f\left[\begin{array}{l}
\tan \left(\psi_{Y}\right) \\
\tan \left(\psi_{X}\right) \\
-1
\end{array}\right]
$$

Where, $\left(x_{0}, y_{0}\right)$ are the image coordinates of the principal point.

At last, replacing the $\tan \left(\psi_{X}\right)$ and $\tan \left(\psi_{Y}\right)$ in Equation 1 by the $x-x_{0}$ and $y-y_{0}$ in Equation 2 and the reverse form of the Equation 1, which transforms from the object space to image space, can be expressed as:

$$
\begin{aligned}
& x-x_{0}=-f \frac{a_{11}\left(X_{P}-X_{S}(t)\right)+a_{12}\left(Y_{P}-Y_{S}(t)\right)+a_{13}\left(Z_{P}-Z_{S}(t)\right)}{a_{31}\left(X_{P}-X_{S}(t)\right)+a_{32}\left(Y_{P}-Y_{S}(t)\right)+a_{33}\left(Z_{P}-Z_{S}(t)\right)} \\
& y-y_{0}=-f \frac{a_{21}\left(X_{P}-X_{S}(t)\right)+a_{22}\left(Y_{P}-Y_{S}(t)\right)+a_{23}\left(Z_{P}-Z_{S}(t)\right)}{a_{31}\left(X_{P}-X_{S}(t)\right)+a_{32}\left(Y_{P}-Y_{S}(t)\right)+a_{33}\left(Z_{P}-Z_{S}(t)\right)}
\end{aligned}
$$

Where, $a_{i j}(i=1,2,3 ; j=1,2,3)$ are elements of the rotation matrix from body coordinate system to the geocentric Cartesian coordinate system.

\subsection{RFM of high resolution satellite imagery}

RFM (Tao and $\mathrm{Hu}, 2001$ ) performs the same transformation through a generalized version of polynomials, and a ratio of two polynomials, which is similar as co-linearity equation. The RFM can be expressed as following:

$$
r=\frac{P_{1}(U, V, W)}{P_{2}(U, V, W)} \quad c=\frac{P_{3}(U, V, W)}{P_{4}(U, V, W)}
$$

Where, $(r, c)$ are the normalized image coordinates to the range from -1.0 to 1.0 by their image size, $(U$, $V, W)$ are the normalized ground coordinates to the range from -1.0 to 1.0 by their geometric extend. $P_{i}(U, V, W) \quad(i=1,2,3$ and 4$)$ are the polynomial.

Usually, the order of the polynomials is limited by $0 \leq \mathrm{m}_{1} \leq 3,0 \leq \mathrm{m}_{2} \leq 3,0 \leq \mathrm{m}_{3} \leq 3$ and $\mathrm{m}_{1}+\mathrm{m}_{2}+\mathrm{m}_{3} \leq 3$. Each $P_{i}(U, V, W) \quad(i=1,2,3$ and 4$)$ is then a third order twenty term polynomial:

$$
\begin{aligned}
& P_{i}(U, V, W)=a_{0}+a_{1} U+a_{2} V+a_{3} W+a_{4} U V+a_{5} U W+a_{6} V W+ \\
& a_{7} U^{2}+a_{8} V^{2}+a_{9} W^{2}+a_{10} U V W+a_{11} U^{2} V+a_{12} U^{2} W+a_{13} U V^{2}+ \\
& a_{14} V^{2} W+a_{15} U W^{2}+a_{16} V W^{2}+a_{17} U^{3}+a_{18} V^{3}+a_{19} W^{3}
\end{aligned}
$$

If equation (4) is substituted in Equation (3) and eliminated the first coefficient in the denominator, the RFM becomes: 


$$
\left\{\begin{array}{c}
r=\frac{\left(1, U, V, W, \cdots, U^{3}, V^{3}, W^{3}\right)\left(a_{0}, a_{1}, a_{2}, \cdots, a_{19}\right)^{T}}{\left(1, U, V, W, \cdots, U^{3}, V^{3}, W^{3}\right)\left(1, b_{1}, b_{2}, \cdots, b_{19}\right)^{T}} \\
c=\frac{\left(1, U, V, W, \cdots, U^{3}, V^{3}, W^{3}\right)\left(c_{0}, c_{1}, c_{2}, \cdots, c_{19}\right)^{T}}{\left(1, U, V, W, \cdots, U^{3}, V^{3}, W^{3}\right)\left(1, d_{1}, d_{2}, \cdots, d_{19}\right)^{T}}
\end{array}\right.
$$

Where, $a_{i} \quad(i=0, \cdots, 19)$ are RPCs.

\subsection{RPC generation of ZY-3 imagery}

\subsubsection{Virtual grid corresponding points generation}

The virtual grid corresponding points (VGCPs) were generated to estimate the RPCs by employing the terrain-independent approach (Figure 3), which depends upon the RSM of ZY-3 imagery. Terrain-independent approach was used to generate the VGCPs from object space to image space. This conversion includes three steps as following:

(1) The object space was divided into $\mathrm{m} \times \mathrm{n}$ grids in the first step;

(2) And then, the corresponding elevation of object grid points can be obtained from the ASTER G-DEM $(30 \mathrm{~m})$ covering the full extent of the image;

(3) To calculate the image grid coordinates of corresponding object grid points in accordance with the linear array co-linearity Equation 3 using VGCPs in image space for ZY-3 imagery.

And then, half VGCPs were selected as control points (CTPs) to estimate the RPCs. At the same time, the remaining half VGCPs as check points (CKPs) were used to evaluate the RPC generation accuracy.

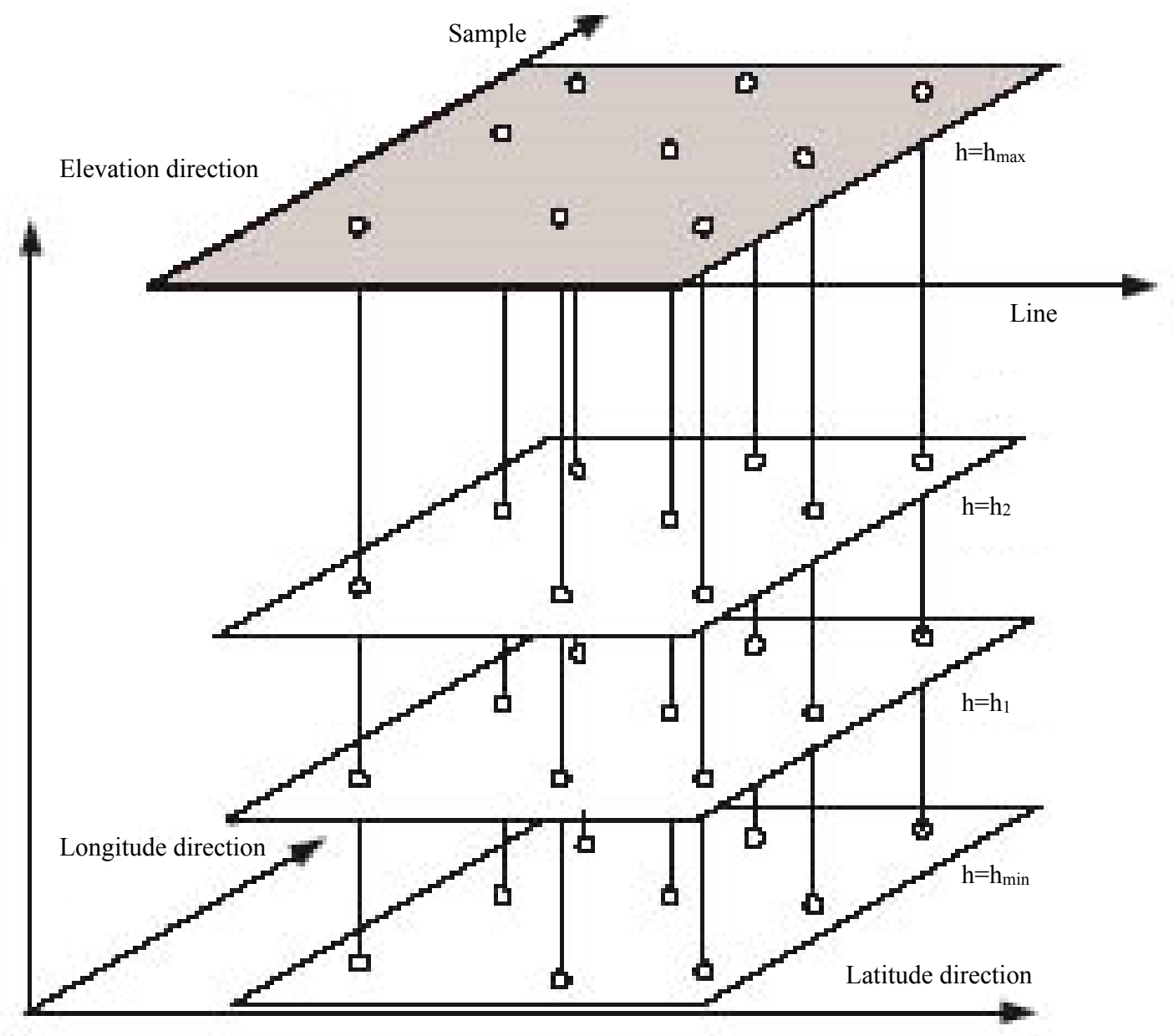

Figure 3. Virtual control grid construction based on independent terrain (Fraser et al., 2006) 


\subsubsection{RPCs estimation}

The 78 unknown RPCs are then can be estimated by least squares adjustment according by the VGCPs corresponding generated by method introduced in Section 3.3.1.

After linearlizing from the Equation 6, we have:

$$
\left\{\begin{array}{l}
D_{1} v_{r}=\left(1, U, \cdots, W^{3},-r U, \cdots,-r W^{3}\right) \cdot\left(a_{0}, a_{1}, \cdots, a_{19}, b_{1}, \cdots, b_{19}\right)^{T}-r \\
D_{2} v_{c}=\left(1, U, \cdots, W^{3},-c U, \cdots,-c W^{3}\right) \cdot\left(c_{0}, c_{1}, \cdots, c_{19}, d_{1}, \cdots, d_{19}\right)^{T}-c
\end{array}\right.
$$

Where,

$$
\left\{\begin{array}{l}
D_{1}=\left(1, U, V, W \cdots, U^{3}, V^{3}, W^{3}\right) \cdot\left(1, d_{1}, d_{2}, \cdots, d_{19}\right)^{T} \\
D_{2}=\left(1, U, V, W \cdots, U^{3}, V^{3}, W^{3}\right) \cdot\left(1, b_{1}, b_{2}, \cdots, b_{19}\right)^{T}
\end{array}\right.
$$

Since there are 78 unknown coefficients in Equation 8, more than 39 CTPs with known image and ground coordinates are needed to have a least squares solution.

The above observation equation can also be expressed in matrix form as:

$$
V=A X-L
$$

Its least squares solution is

$$
\hat{X}=\left(A^{T} A\right)^{-1}\left(A^{T} L\right)
$$

\subsubsection{RPCs validation}

Estimated RPCs is then used to calculate the 2D image coordinates of the CKPs selected from generated VGCPs. By calculating the difference between the 2D image coordinates of CKPs calculated from the RPCs and those calculated from the RSM, the accuracy of the RPCs generation can be evaluated.

\section{Results and discussion}

In the experiments, RPCs generation for ZY-3 imagery as introduced in Section 3.3 was conducted for three data sets. To evaluate the accuracy and reliability of the proposed method, the experiments have been designed as four aspects. The first aspect is comparing the performance of RPC generation with different terrain features (such as: Ocean Region (Sansha City), city region (Shanghai City) and grassland region (Hulunbuir City)). The second aspect is comparing the performance of RPC generation with different sensor (such as: forward camera, nadir camera and backward camera). The third aspect is evaluating the influence on the size of the grid of VGCPs. The fourth aspect is evaluating the influence on the number of height layers of VGCPs.

To ensure adequate number of observations, the minimum size of VGCPs is defined as $10 \times 10$ (10 lines by 10 samples in a horizontal plane) with at least 3 elevation layers, These scenarios are using different sizes from 10 $\times 10$ to $30 \times 30$ in line and sample direction and different sizes from 3 to 10 in elevation direction to evaluate the performance of different sizes of VGCPs.

\subsection{Comparison of the performance of RPCs generation with different terrain features}

Figure 4 shows the accuracy in both $\mathrm{x}$ direction and $\mathrm{y}$ direction in image space of RPCs generation for ZY-3 three-line array imageries in Shanghai region. From the results demonstrated in Figure 6, the RMS error at CKPs was better than 1.144E-04 pixels in line and 1.946E-04 pixels in sample, respectively. Figure 5 shows the accuracy in both $\mathrm{x}$ direction and $\mathrm{y}$ direction in image space of RPCs generation for ZY-3 three-line array imageries in Sansha region. From the results displayed in Figure 5, we see that the RMS error at CKPs is better than 6.555E-05 pixels in line and 1.895E-04 pixels in sample, respectively. Figure 6 shows the accuracy in both $x$ direction and $y$ direction in image space of RPCs generation for ZY-3 three-line array imageries in Hulunbuir region. From the results illuminated in Figure 6, we see that the RMS error at CKPs is better than 1.355E-04 pixels in line and 1.866E-04 pixels in sample, respectively. The results showed in Figure 4-6 proved that the proposed RPCs generation 
approach had achieved high accuracy (better than 2.0 E-04 in both line and sample) which satisfies the requirement of horizontal and vertical accuracy for 1:50000 scale mapping in China, and it revealed that RFM can be widely used to replace the RSM for ZY-3 three-line imageries in ocean region, city region and grassland region, etc.

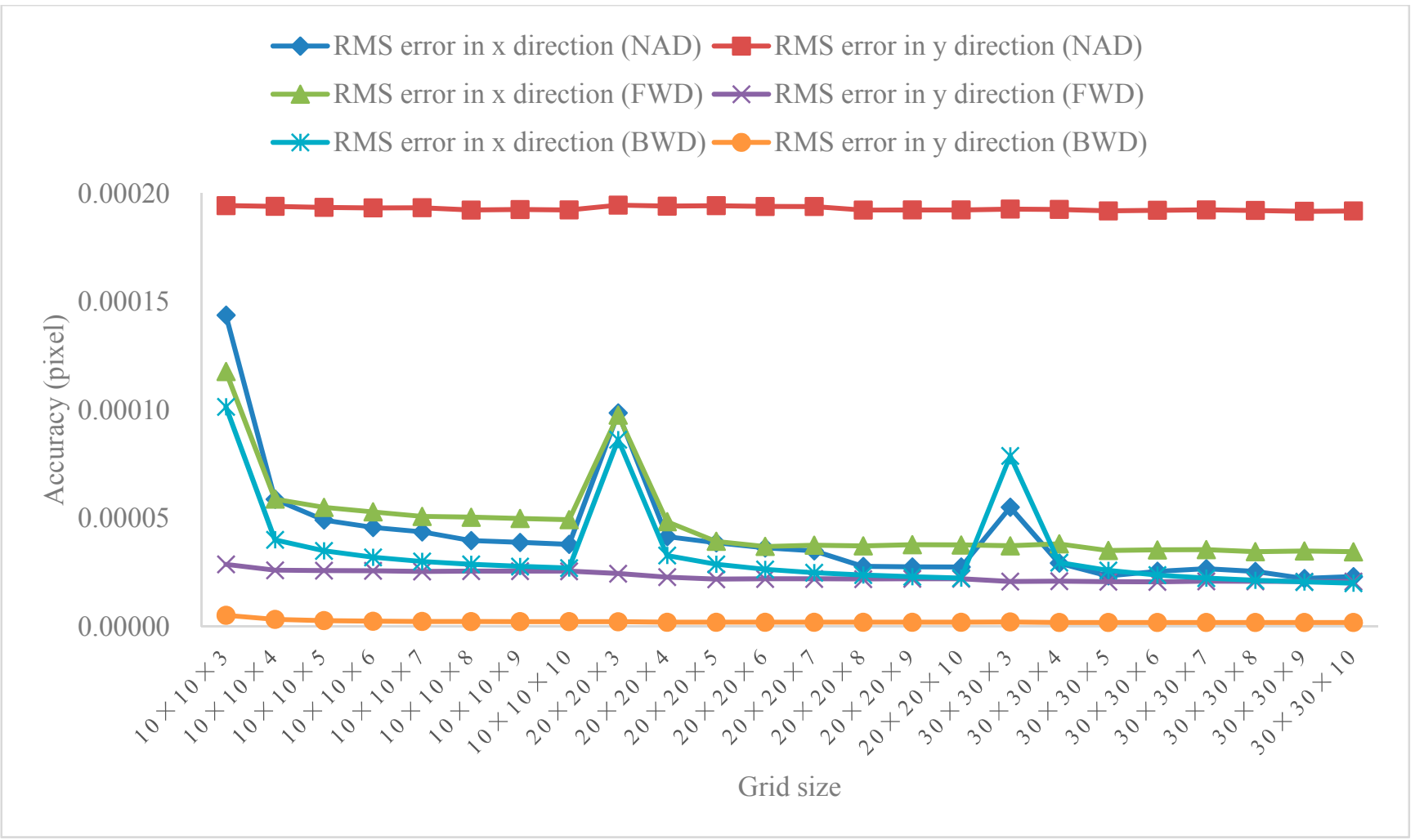

Figure 4. Accuracy of RPCs generation for ZY-3 image in Shanghai study region

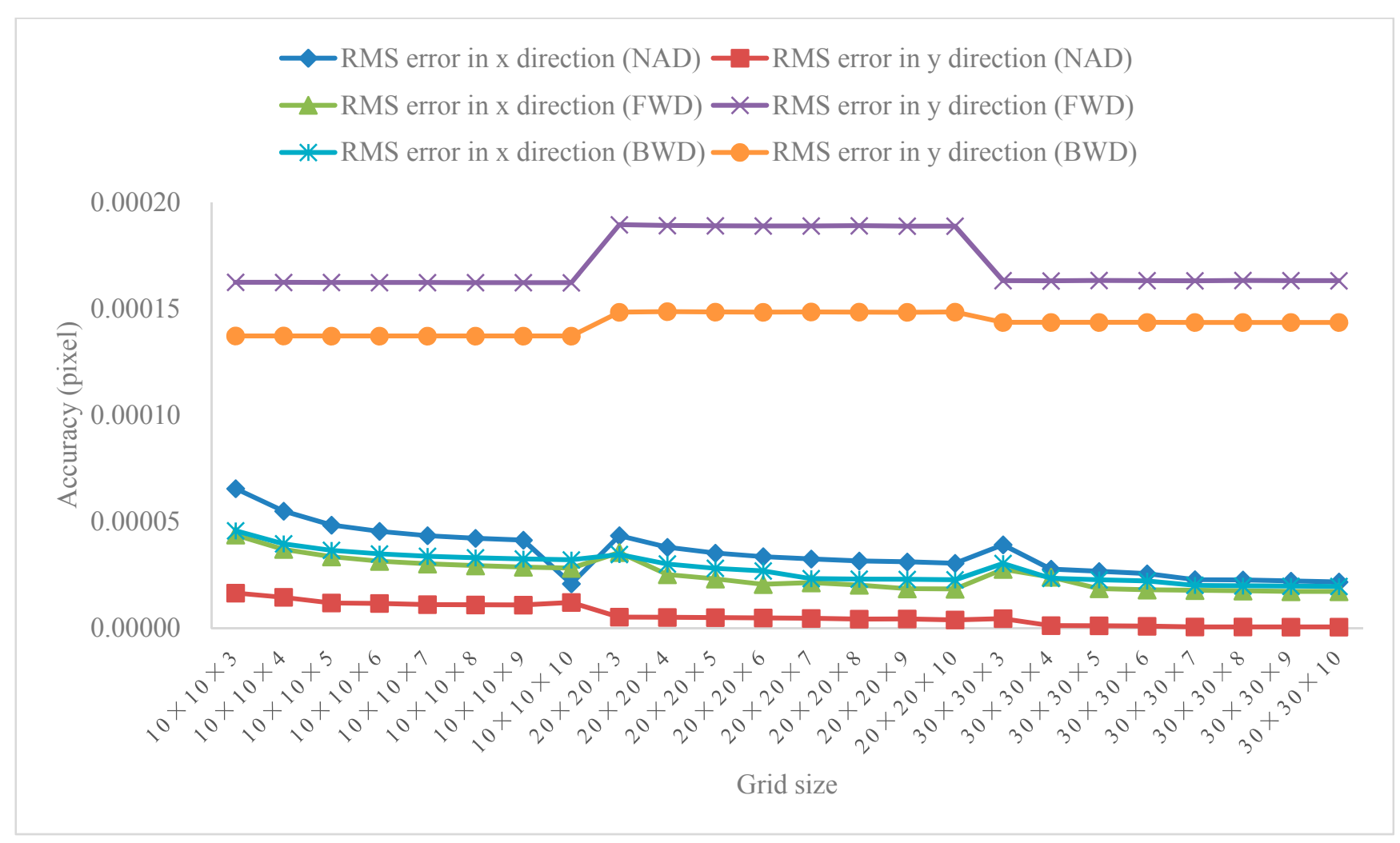

Figure 5. Accuracy of RPCs generation for ZY-3 image in Sansha study region 


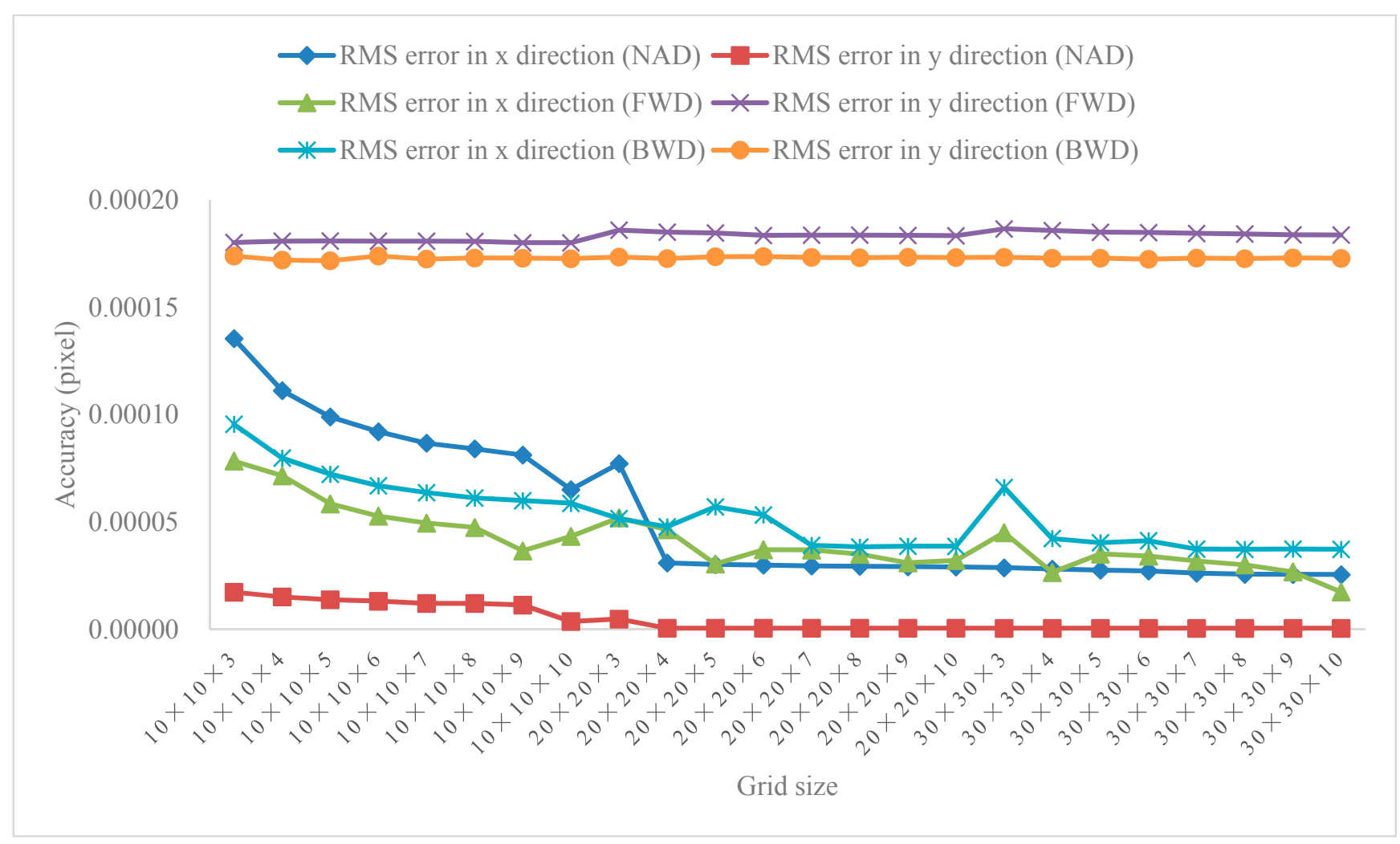

Figure 6. Accuracy of RPCs generation for ZY-3 image in Hulunbuir study region

\subsection{Comparison of the performance of RPCs generation with different sensor image}

Figure 7 and Figure 8 show the comparison of the performance of RPCs generation for ZY-3 forward-nadirbackward imageries in $x$ direction and y direction with $10 \times 10$ in line and sample direction and different sizes from 3 to 10 in elevation direction, respectively. As can be seen from Figure 7 and Figure 8, the accuracy of RPC fitting for ZY-3 backward imagery is better than forward imagery and nadir imagery in $x$ direction. At the same time, the forward imagery and backward imagery have a reasonable consistency and similar trend in both $x$ direction and y direction.
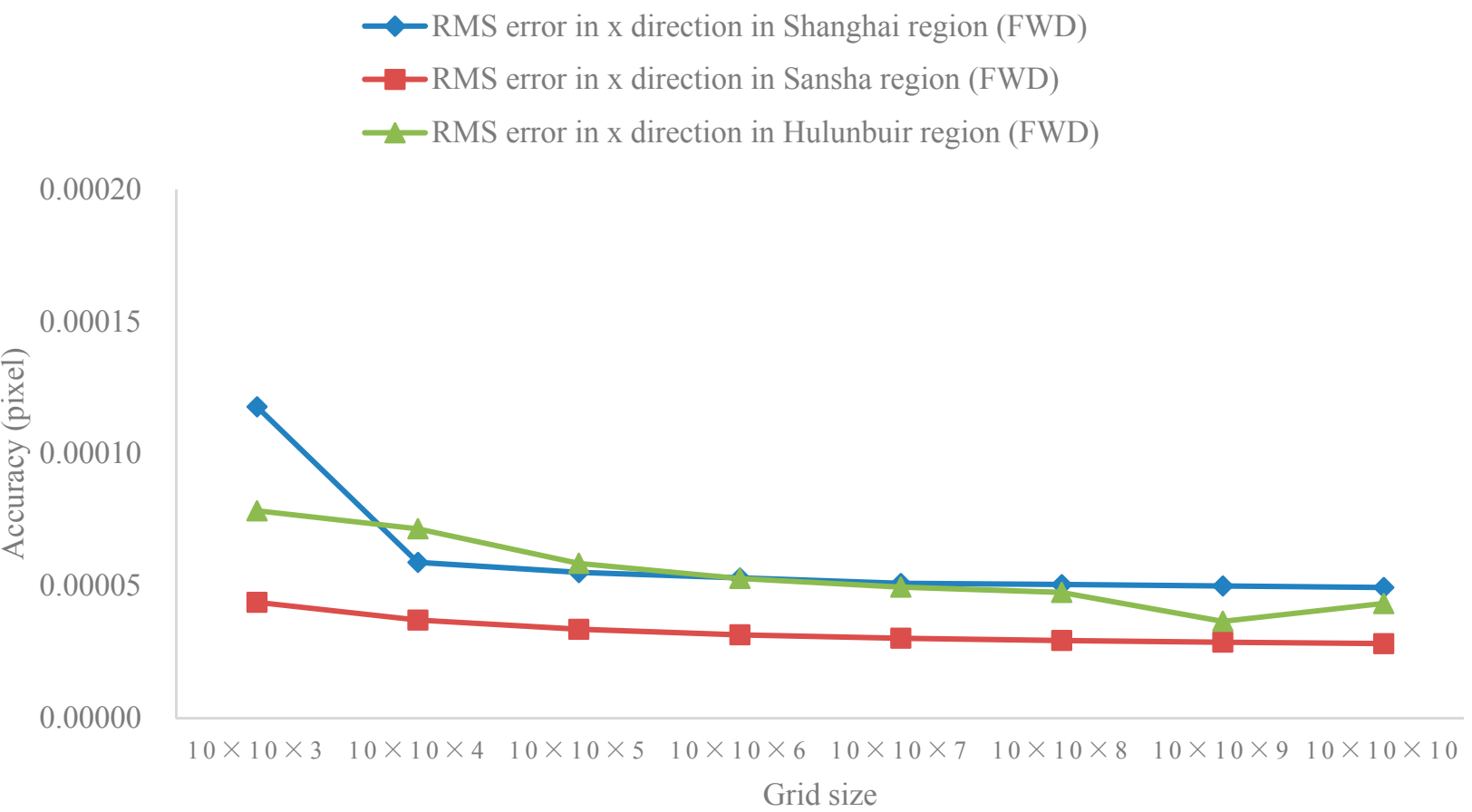
(a) Forward sensor

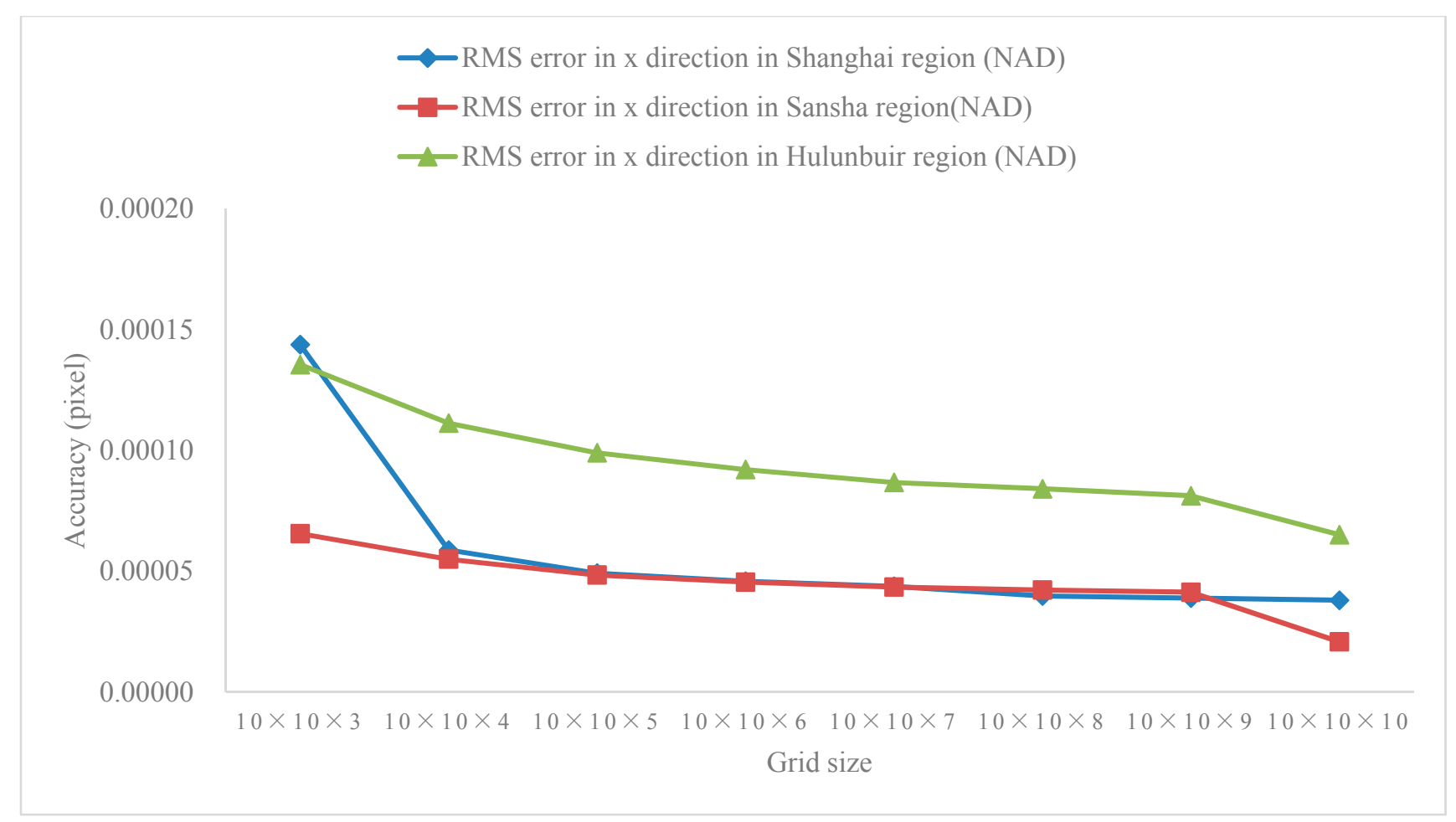

(b) Nadir sensor

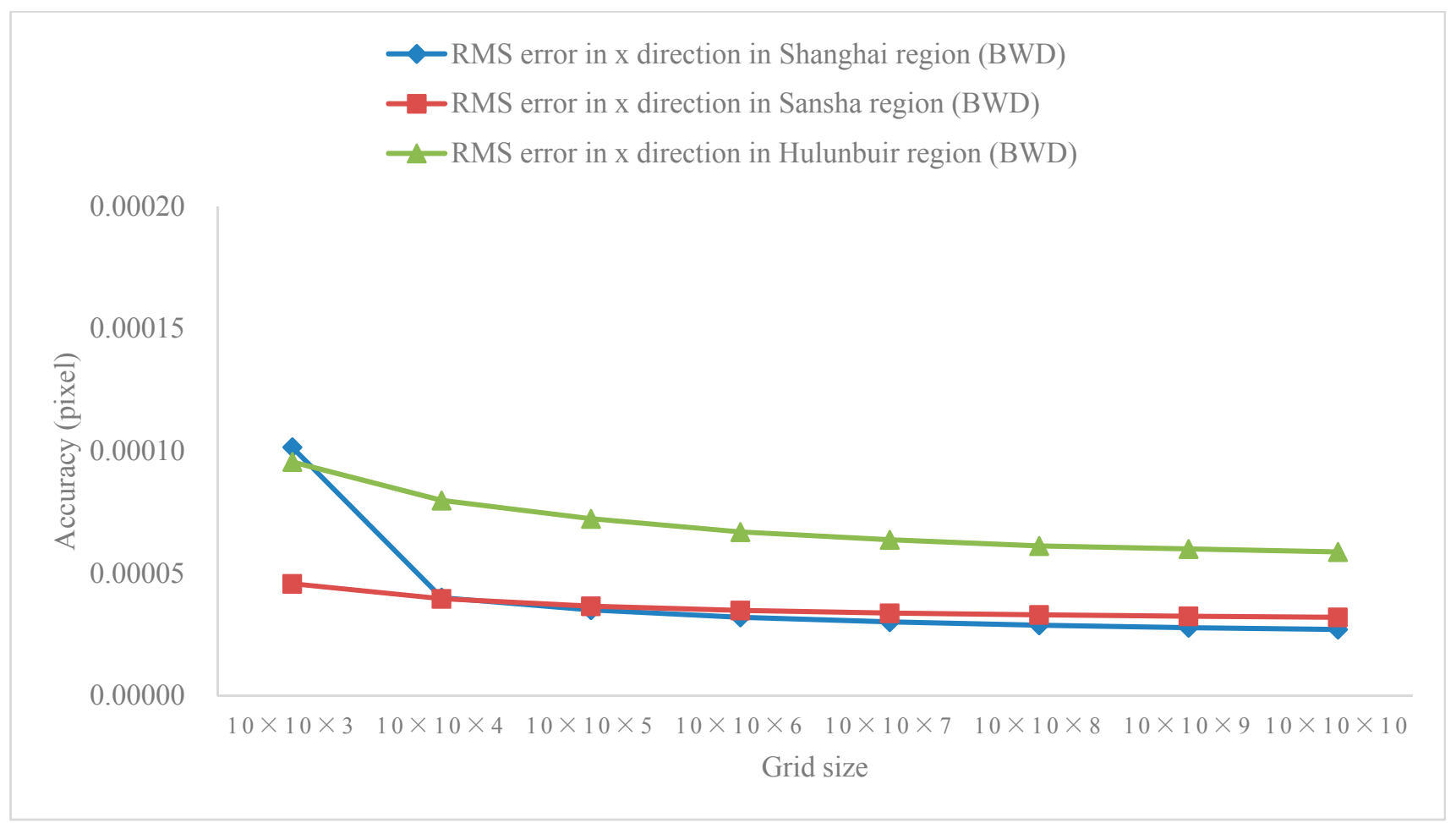

(c) Backward sensor

Figure 7. Accuracy of RPCs generation for ZY-3 forward-nadir-backward images in $\mathrm{x}$ direction 


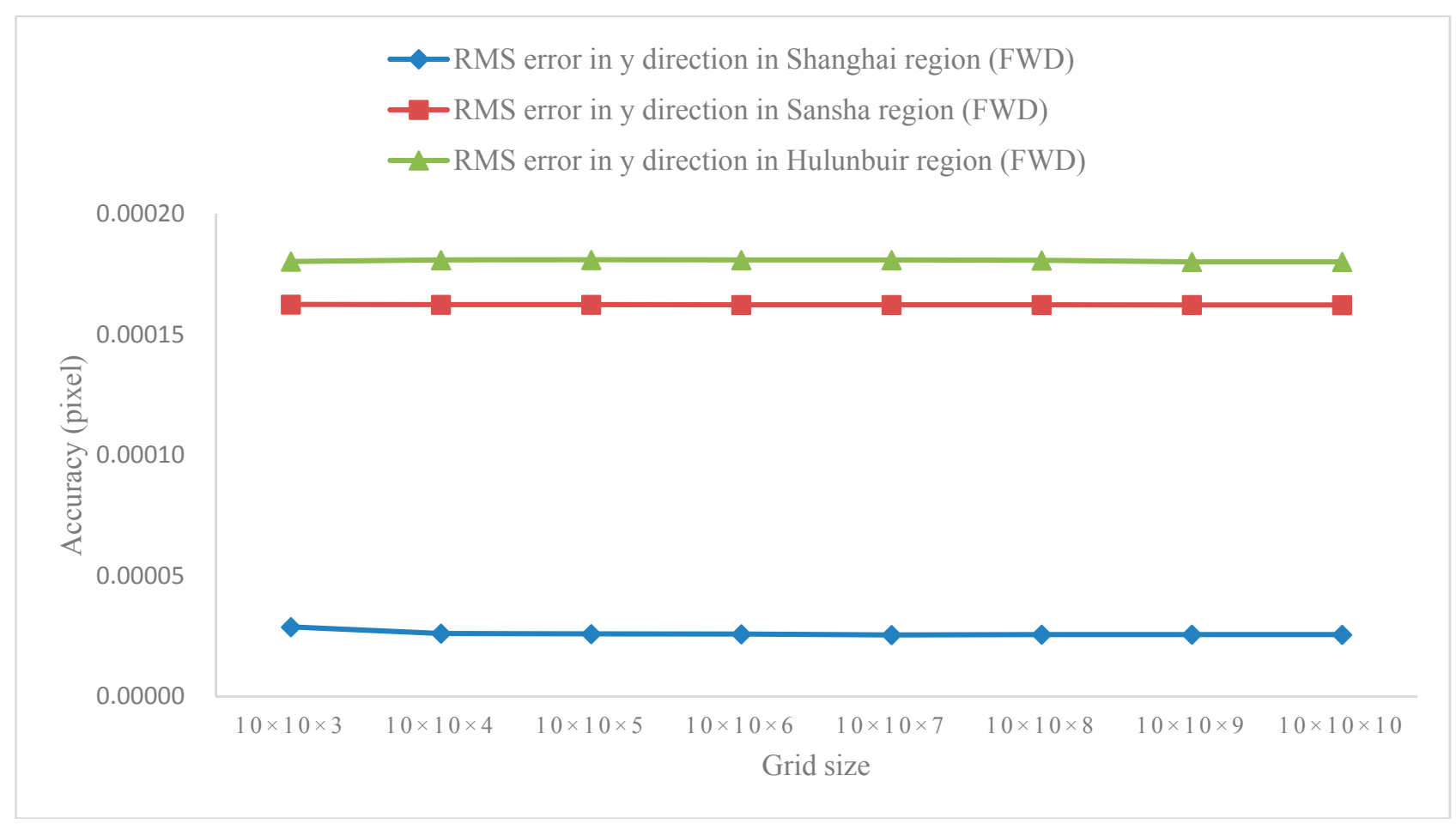

(a) Forward sensor

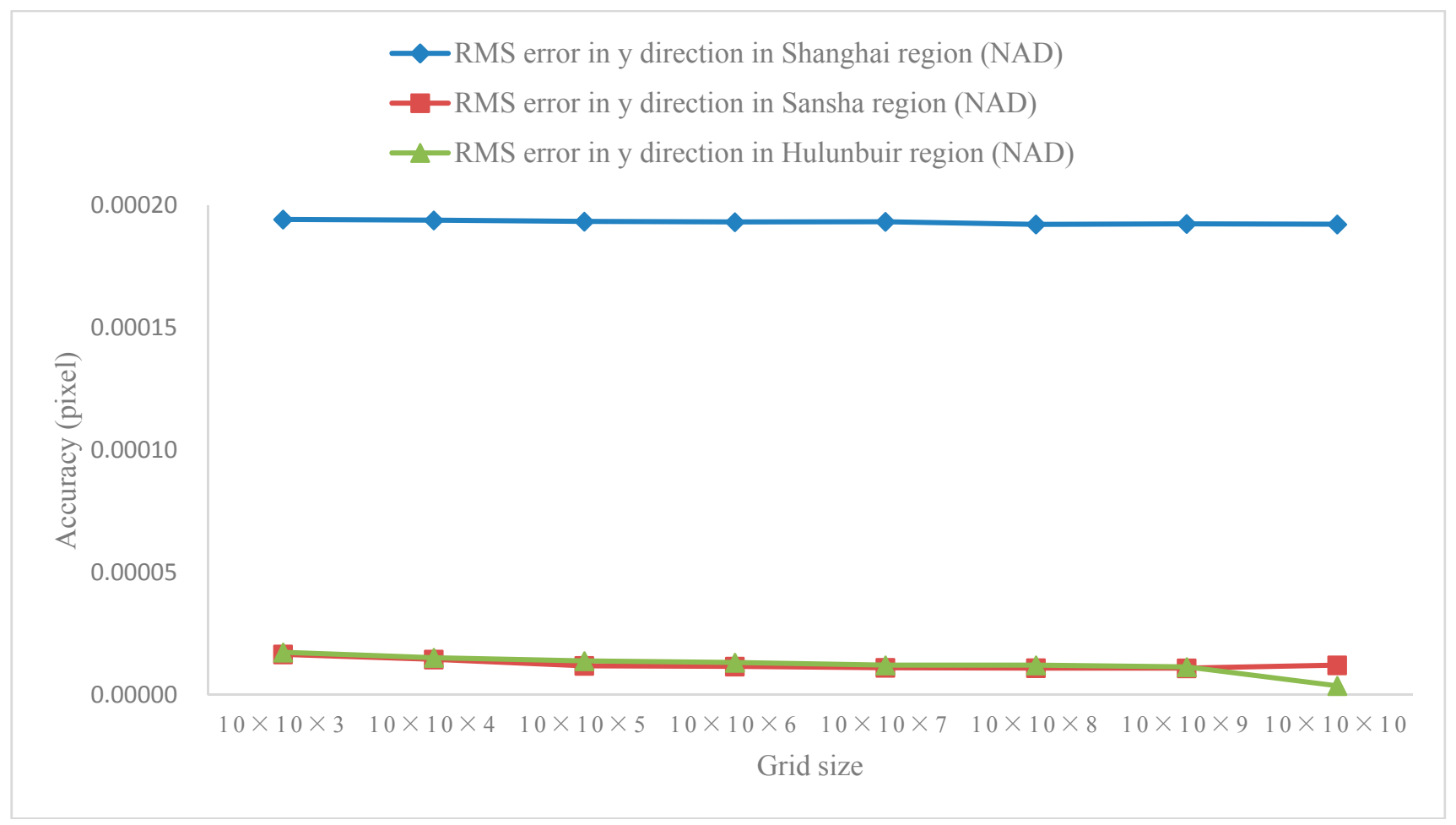

(b) Nadir sensor 


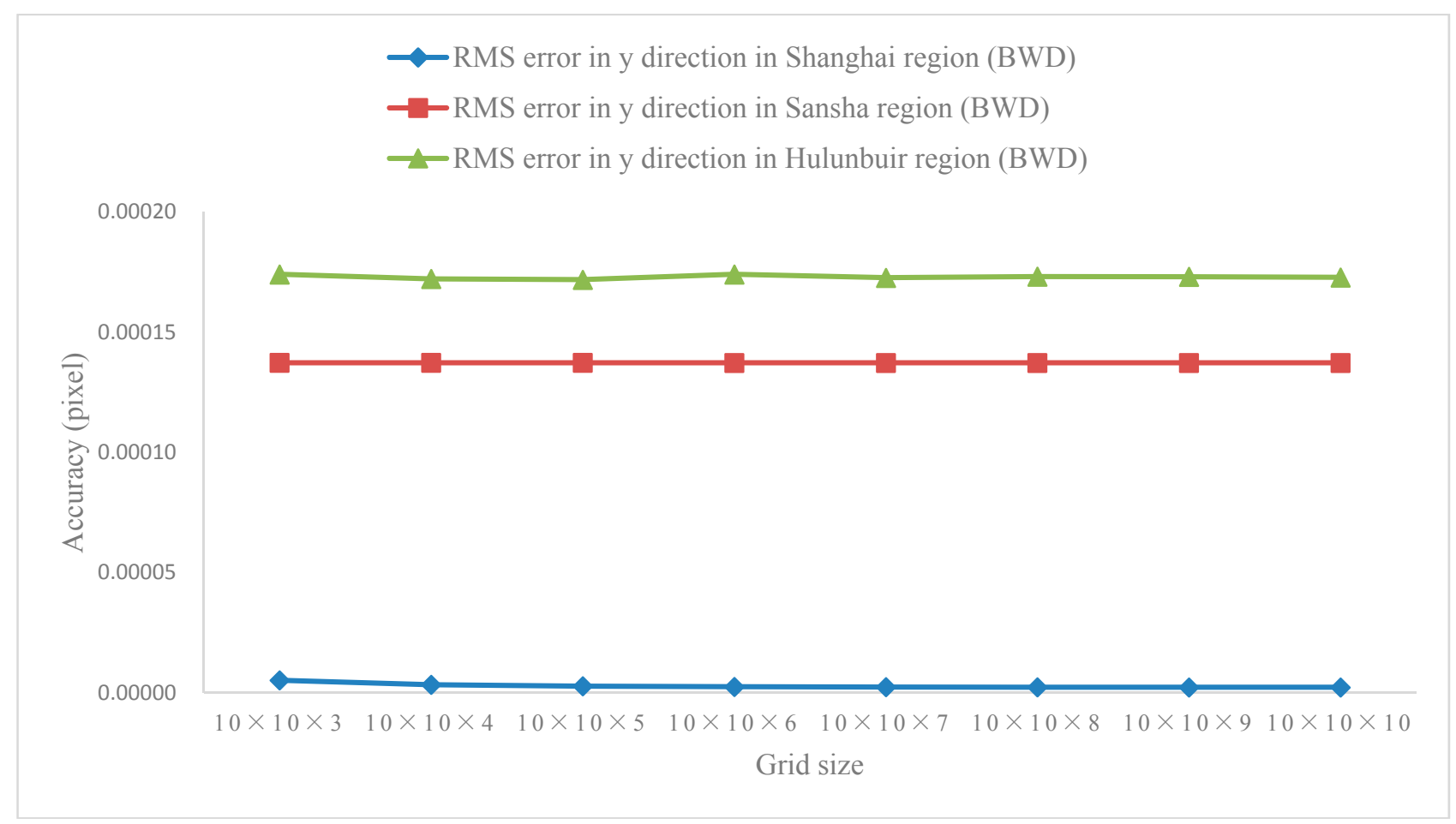

(c) Backward sensor

Figure 8. Accuracy of RPCs generation for ZY-3 forward-nadir-backward images in y direction

\subsection{Comparison of the performance of RPCs generation with different height layers}

In order to evaluate the influence of the height layer on the RPC accuracy, 3D control grids consisting of $10 \times 10$ grid each with height layer from 3 to 10 were established. Figure 9 show the comparison of the performance of different height layers in three study regions in $\mathrm{x}$ direction and y direction, respectively. From the test results, it can be seen that the RMS error of CKPs is reducing as the height layer increases in all test data sets in $x$ direction. Meanwhile, the RMS error of CKPs will not be improved much once the height layer increases in y direction, which means using a denser grid would not be beneficial to accuracy. 


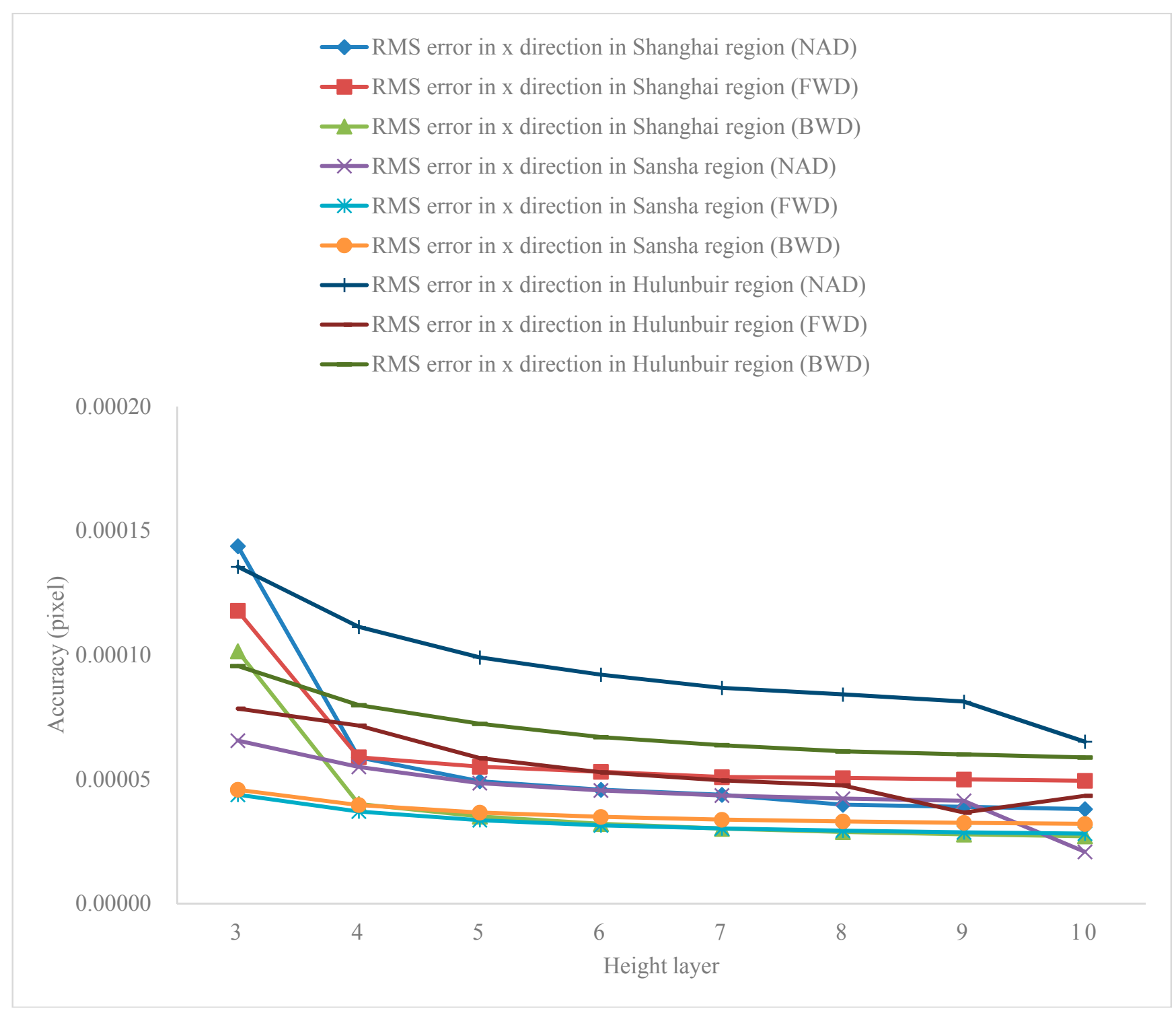

(a) $x$ direction 


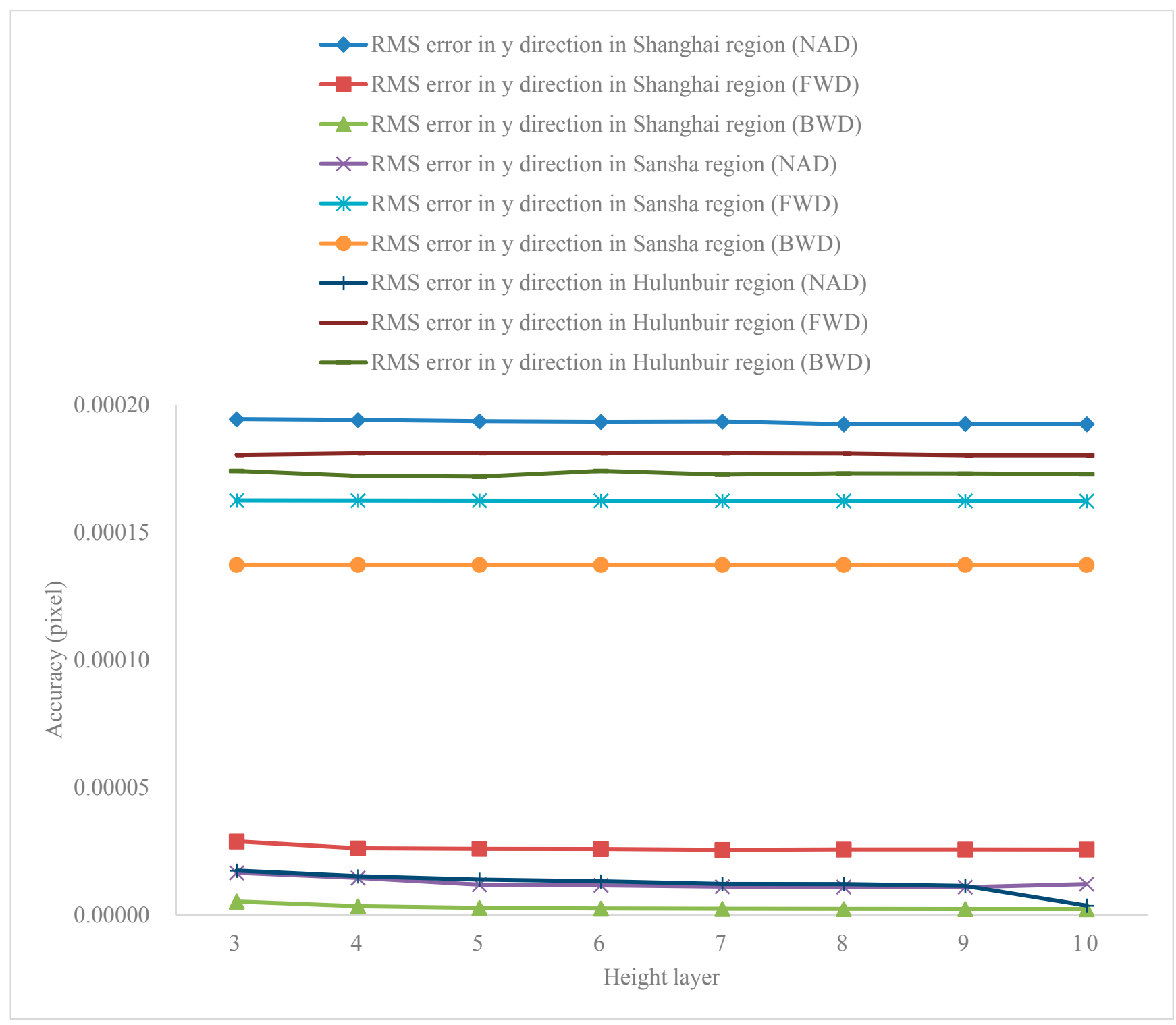

(b) y direction

Figure 9. The accuracy of the CKPs with the height layer on ZY-3 three-line array imageries in both $x$ direction and $y$ direction

\subsection{Comparison of the performance of RPCs generation with different grid sizes}

In order to evaluate the influence of the size of the grid on the RPC accuracy, 3D control grids consisting of three elevation layers each with $10 \times 10$ pixels, $20 \times 20$ pixels, $30 \times 30$ pixels were established. Figure 10 shows the comparison of the performance of different grid sizes in three study regions in $\mathrm{x}$ direction and $\mathrm{y}$ direction, respectively. From the test results, it can be seen that the RMS error of CKPs is reducing as the grid number increases in all test data sets in $x$ direction. Meanwhile, the RMS error of CKPs will not be improved much once the size of grid number increases in y direction, which means using a denser grid would not be beneficial to accuracy. 


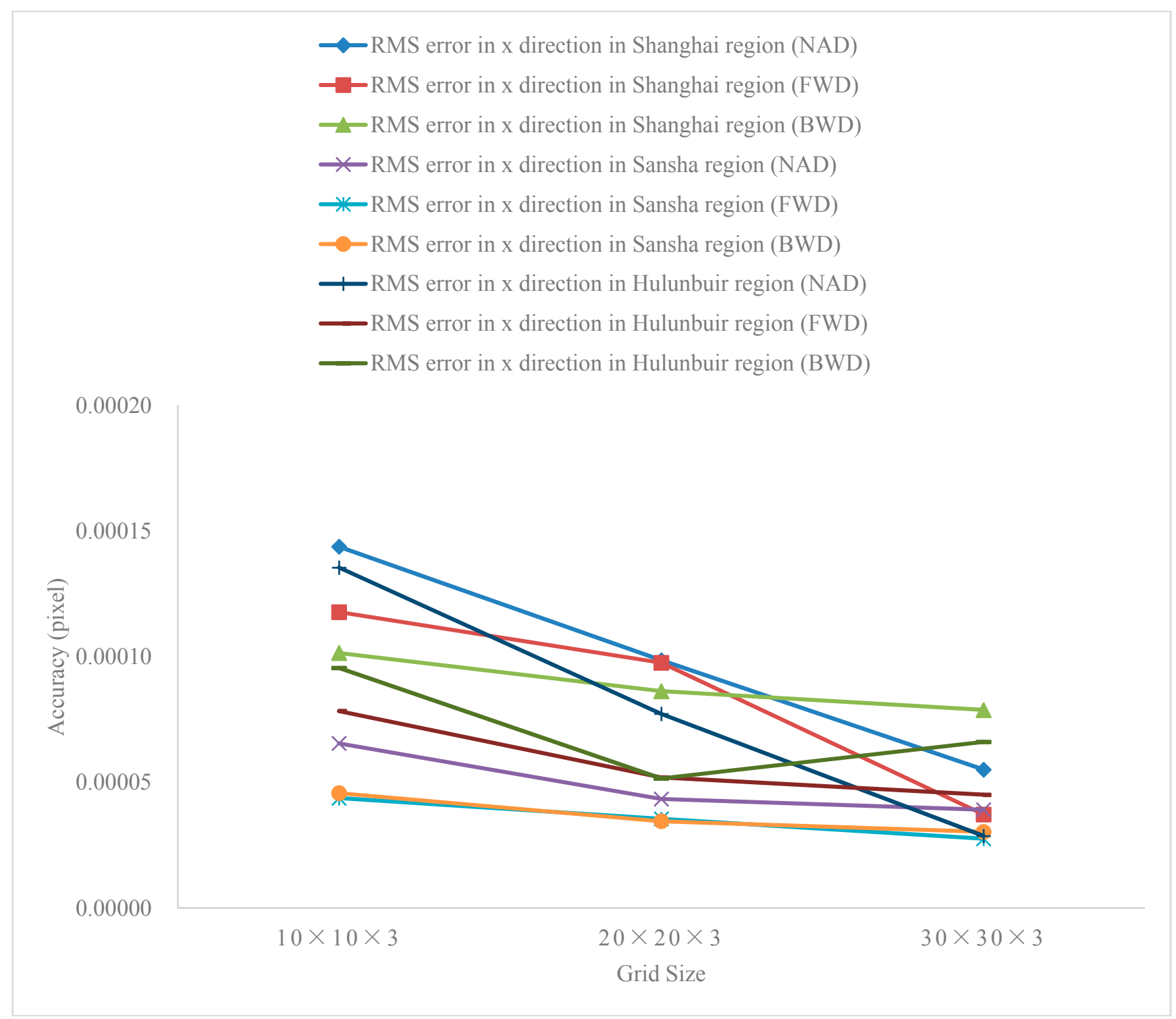

(a) $x$ direction 


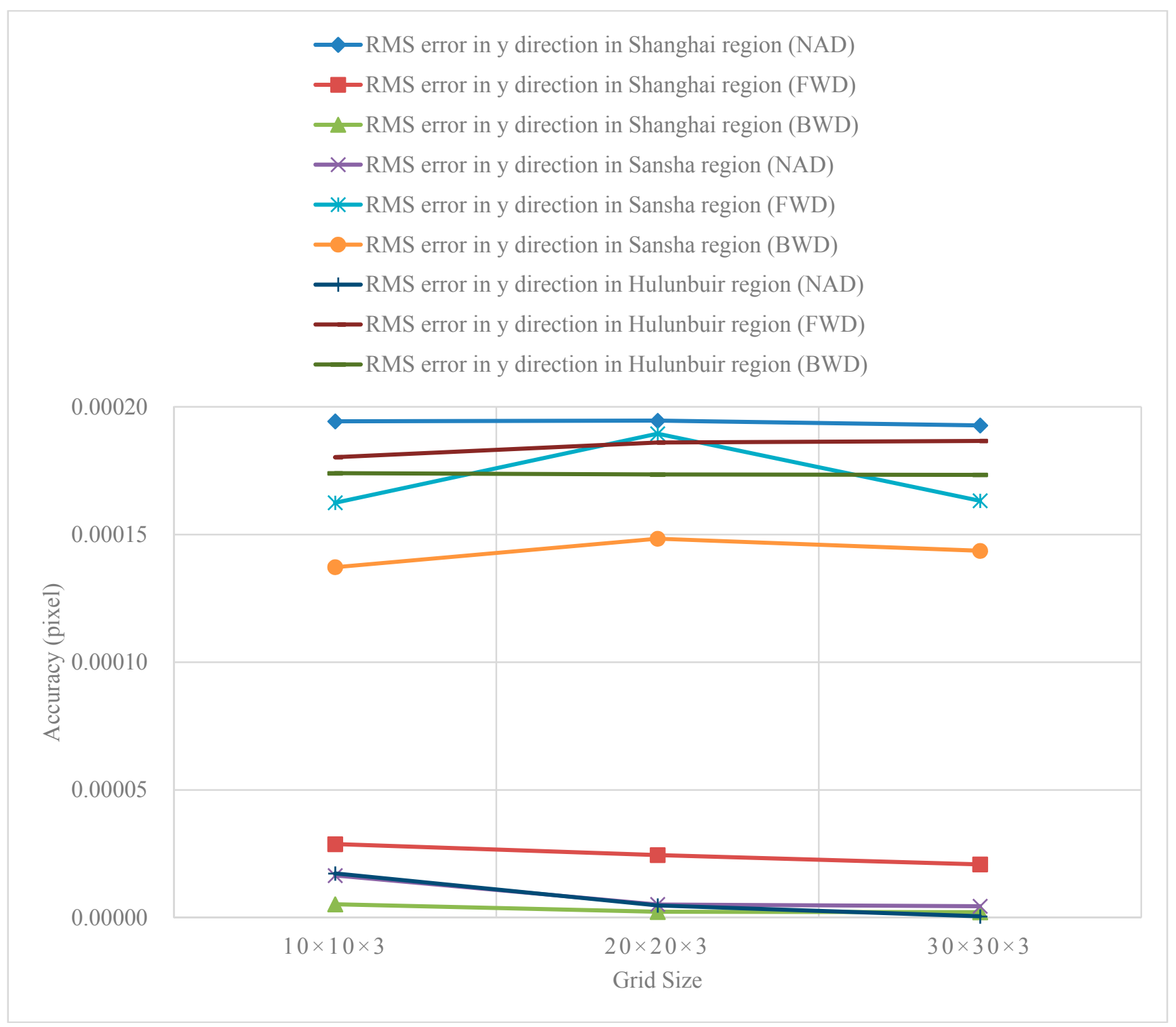

(b) y direction

Figure 10. The accuracy of the CKPs with the grid number on ZY-3 three-line array imageries in both $\mathrm{x}$ direction and $\mathrm{y}$ direction

\section{Conclusions}

This paper has evaluated the feasibility and effectiveness of the proposed method RPCs generation for ZY-3 three-line imageries. Based on three scenarios experiments with different terrain features (such as ocean, city and grassland), several conclusions are drawn as follows:

(1) The RFM model can fit ZY-3 three-line imageries very well and achieve encouraging accuracy of better than 1.946E-04 pixel in both $x$ direction and $y$ direction, and it reveals that RFM can be widely used to replace the RSM for ZY-3 three-line imageries in ocean region, city region and grassland region, etc.

(2) The accuracy of RPCs fitting for ZY-3 backward imagery is better than forward imagery and nadir imagery in $x$ direction. At the same time, the forward imagery and backward imagery have a reasonable consistency and similar trend in both $x$ direction and $y$ direction.

(3) The accuracy of RPCs fitting for ZY-3 three-line imageries is reducing as the grid number increases in all test data sets in $\mathrm{x}$ direction. However, the grid number increases will be not beneficial to accuracy of RPCs fitting when grid number increases in y direction.

(4) The accuracy of RPCs fitting for ZY-3 three-line imageries is performed similarly as grid number when height number of elevation increases in both $\mathrm{x}$ direction and $\mathrm{y}$ direction. 
Acknowledgements: The work described in this paper was substantially supported by the National Natural Science Foundation of China (Project No. 41401489, 41376178), Shanghai Foundation for University Youth Scholars (Project No. ZZHY13033), Innovation Program of Shanghai Municipal Education Commission (Project No. 15ZZ082).

Author Contributions: The concept and design of the framework for RPCs generation were proposed by Zhonghua Hong. The experiments for ZY-3 satellite imagery performed by Shengyuan Xu, Yun Zhang, Yanlin Han and Yongjiu Feng. All authors have contributed significantly and have participated sufficiently to take the responsibility for this research. Moreover, all authors are in agreement with the submitted and accepted versions of the publication and declare no conflict of interest.

\section{References}

1. Chen, Y.; Xie, Z.; Qiu, Z.; Zhang, Q.; Hu, Z. Calibration and validation of ZY-3 optical sensors. IEEE Trans. Geosci. Remote Sens.2015, 53(8), 4616-4626.

2. Dial, G., Bowen, H., Gerlach, F., Grodecki, J., Oleszczuk, R. IKONOS satellite, imagery, and products. Remote Sens Environ, 2003,88(1), 23-36.

3. Eftekhari, A., Saadatseresht, M., Motagh, M. A study on rational function model generation for TerraSAR-X imagery. Sensors, 2013, 13(9), 12030-12043.

4. Fraser, C.S.; Hanley, H.B. Bias compensation in rational functions for Ikonos satellite imagery. Photogramm. Remote Sens. 2003, 69, 53-57.

5. Fraser, C.S.; Hanley, H.B. Bias-compensated RFMs for sensor orientation of high-resolution satellite imagery. Photogramm. Remote Sens. 2005, 71, 909-915.

6. Fraser, C.S.; Dial, G.; Grodecki, J. Sensor orientation via RPCs. ISPRS J. Photogramm. Remote Sens. 2006, 60, 182-194.

7. Fraser, C.S.; Ravanbakhsh, M. Georeferencing accuracy of GeoEye-1 imagery. Photogramm. Remote Sens. 2009, 75, 634-638.

8. Jannati, M., Valadan Zoej, M. J., Mokhtarzade, M. Epipolar Resampling of Cross-Track Pushbroom Satellite Imagery Using the Rigorous Sensor Model. Sensors, 2017,17(1), 129,1-19.

9. Li, R.; Zhou, F.; Niu, X.; Di, K. Integration of IKONOS and QuickBird imagery for geopositioning accuracy analysis. Photogramm. Remote Sens. 2007, 73, 1067-1074.

10. Li, R.; Deshpande, S.; Niu, X.; Zhou, F.; Di, K.; Wu, B. Geometric integration of aerial and high-resolution satellite imagery and application in shoreline mapping. Mar Geod. 2008, 31, 143-159.

11. Poli, D. A rigorous model for spaceborne linear array sensors. Photogramm. Remote Sens. 2007, 73, 187-196.

12. Poli, D., Toutin, T. Review of developments in geometric modelling for high resolution satellite pushbroom sensors. Photogramm Rec, 2012, 27(137), 58-73.

13. Poli D, Remondino F, Angiuli E, Agugiaro, G. Radiometric and geometric evaluation of GeoEye-1, WorldView-2 and Pléiades-1A stereo images for 3D information extraction. ISPRS J. Photogramm. Remote Sens, 2015, 100, 35-47.

14. Tang, X., Zhou, P., Zhang, G., Wang, X., Pan, H. Geometric accuracy analysis model of the ZiYuan-3 satellite without GCPs. Photogramm. Remote Sens, 2015, 81(12), 927-934.

15. Tao, C.V.; Hu, Y. A comprehensive study of the rational function model for photogrammetric processing. Photogramm. Remote Sens. 2001, 67, 1347-1357.

16. Toutin, T. Review article: geometric processing of remote sensing images: models, algorithms and methods. INT J Remote Sen. 2004, 25, 1893-1924.

17. Tong, X.H.; Liu, S.J.; Weng, Q.H. Bias-corrected rational polynomial coefficients for high accuracy geo-positioning of QuickBird stereo imagry. ISPRS J. Photogramm. Remote Sens. 2010, 65, 218-226.

18. Tong, X.H.; Hong, Z.H.; Liu, S.J; Zhang, X.; Xie, H.; Li, Z.Y.; Yang, S.L.; Wang, W.A.; Bao, F. Building-damage detection using pre- and post-seismic high-resolution IKONOS satellite stereo imagery: a case study of the May 2008 Wenchuan Earthquake. ISPRS J. Photogramm. Remote Sens. 2012, 68, 13-27. 